\title{
APOYO SOCIAL Y SENTIDO DE COMUNIDAD EN DESPLAZADOS Y DAMNIFICADOS EN EL DEPARTAMENTO DEL MAGDALENA
}

\section{SOCIAL SUPPORT AND SENSE OF COMMUNITY IN DISPLACED PEOPLE AND VICTIMS IN THE DEPARTMENT OF MAGDALENA}

\section{TITULO CORTO: APOYO SOCIAL Y SENTIDO DE COMUNIDAD EN DESPLAZADOS Y DAMNIFICADOS}

\section{Aníbal Camargo-Velásquez ${ }^{1}$, Jorge Palacio-Sañudo²}

Recibido en febrero 19 de 2016

Aceptado en mayo 23 de 2016

Financiación: Gobernación del Magdalena, a través del Fondo de Ciencia Tecnología e Innovación del Sistema General de Regalías.

\section{RESUMEN}

En esta investigación se correlacionan las variables sentido de comunidad y apoyo social, en un grupo de 136 personas en situación de desplazamiento por la violencia; y víctimas de desastres de la ola invernal del 2010-2011 en el departamento del Magdalena. Los instrumentos utilizados fueron, la Escala de Sentido de Comunidad y el Cuestionario de apoyo social-MOS (Estudio de Desenlaces Médicos de Apoyo Social), adaptado para Colombia. Para el análisis de los datos, los grupos se dividieron en dos: el primero con 48 hombres entre 26 y 89 años (media de 57 años) y 36 mujeres entre 23 y 87 años (media de 55 años). En el segundo grupo participaron 44 hombres entre 25 y 91 años (media de 58 años) y 8 mujeres entre 23 a 49 años (media de 36 años). En cuanto al índice de sentido de comunidad se halló que el nivel es medio-alto $(\mathrm{m}=53,42)$, con el apoyo social se encontró que el índice general de apoyo social es alto $(87,41)$. Como resultado dio una relación débil, positiva con un coeficiente de correlación 0,27 y significativa (valor P de 0,01 ). Se concluye que las dos variables interactúan entre sí, pero con una débil relación.

Palabras clave: víctimas de desastres; desplazamiento; apoyo social

\section{ABSTRACT}

This research correlating variables, sense of community and social support, in a group of 136 people that were displaced people by violence; and disaster victims of the winter season 2010-2011 in the department of Magdalena. The instruments used to measure the variables were, the Scale of Sense of Community and the Social Support Questionnaire - MOS (Medical Outcomes Study-Social Support Survey) adapted for Colombia. For the analysis of the data, they were divided into two groups: the first with 48 men between 26 and 89 years (mean 57 years) and 36 
women between 23 and 87 years (mean 55 years). In the second group they involved 44 men between 25 and 91 years (mean 58 years) and 8 women between 23 and 49 years (mean 36 years). As the rate of sense of community it was found that the level is medium-high $(\mathrm{m}=53,42)$, the social support it was found that the overall rate of social support is high $(87,41)$. A weak relationship was found, a positive correlation coefficient 0.27 and significant ( $\mathrm{P}$ value of 0.01 ). We conclude that the two variables interact with each other but with a weak relationship.

Keywords: disaster victims; displacement; social support

\section{INTRODUCCIÓN}

$E^{1}$ desplazamiento forzado y los desastres naturales, han golpeado a la República de Colombia por años, causando alteración de todos los órdenes sociales de las poblaciones afectadas. Los seres humanos como individuos de una sociedad, están siempre a la expectativa que ocurran situaciones que alteren su bienestar y calidad de vida, ya sea por situaciones de índole humana como por ejemplo el desplazamiento forzado o por condiciones naturales como terremotos, huracanes, avalanchas, ola invernal, entre otros.

Tanto los afectados por el fenómeno de la lluvia y victimas por el desplazamiento a causa del conflicto armado, logran de forma negativa tener un rompimiento de vínculos con familiares, amigos y la comunidad, lo cual trae consigo la disminución del apoyo social que perciben las personas ${ }^{1}$, entendiendo que el apoyo social lo componen "las provisiones expresivas o instrumentales - percibidas o reales- proporcionadas por la comunidad, las redes sociales y las relaciones íntimas y de confianza"2.

El apoyo social es fundamental para el desarrollo social y cultural de una comunidad, puesto que garantiza en ésta el fortalecimiento de las relaciones dentro un grupo, en otras palabras, permitirá de forma positiva tener un sentido de comunidad que según Sarason ${ }^{3}$ "es la interdependencia consciente con otros, de tener por voluntad propia esta relación estableciendo sentimientos mutuos que generan una estructura sólida dentro de una comunidad, también se puede expresar que el sentido de comunidad es la responsabilidad entre los miembros de un grupo determinado que se da de manera recíproca, donde no existe el individualismo egoísta"4 y que además de un involucramiento por parte de los miembros de la comunidad se construye el tejido social que promueve el sentido de comunidad ${ }^{5}$.

En el desplazamiento forzado, las personas o grupos de personas son sometidas por medio de la coerción a renunciar a sus hogares y/o tierras como una consecuencia directa de la guerra o conflicto armado dentro del país, vulnerando sus derechos humano ${ }^{6}$ como ciudadanos. En Colombia, el desplazamiento forzado ha sido de mucho interés en las disciplinas de las ciencias sociales y humanas. En plena década del 80, se aumentó el narcotráfico y los grupos al margen de la Ley, hubo incrementos en las cifras por la agudización del conflicto armado, exacerbando las discordias por controles en las tierras y las discrepancias por paradigmas ideológicos y políticos entre paramilitares y guerrilleros, lo que desencadenó vertiginosamente el desplazamiento interno ${ }^{7}$. El desplazamiento forzado es aquel donde la persona o grupo de personas han sido obligadas a desplazarse a otros corregimientos o municipios dentro del territorio nacional o por fuera del país, y como consecuencia se presentan violaciones de los derechos humanos ${ }^{6}$ porque su ser, en cuanto a la integridad física, seguridad o libertad, está siendo amenazado ${ }^{8,9}$ como constante del conflicto armado que está viviendo.

Es así como en el país según expresa Mora $^{10}$ al año son obligadas a salir de sus hogares alrededor de doscientas mil personas, donde el $85 \%$ de los grupos familiares provienen de las zonas rurales y centro poblados demostrando una vez más, que el conflicto armado sigue golpeando con más fuerzas las zonas lejanas de cada municipio y sólo el 15\% de los hogares son de las cabeceras municipales.

En Colombia, las víctimas registradas históricamente hasta corte (01 de abril 2016) han sido 7.999.663 de los 
cuales 6.183 .105 son sujeto de asistencia y reparación ${ }^{11}$. El Departamento de la Prosperidad Social ${ }^{12}$ manifiesta que en Colombia se encuentran registrados aquellos hogares que han sido destituidos de forma individual o masivamente. Esta entidad expresa un orden de éstos, inicialmente se encuentra Antioquia donde hubo un reporte de 131.931 hogares; le sigue Bolívar con 62.528 hogares; en tercer lugar se cuenta con Magdalena, el foco de nuestra investigación, con un reporte de 51.609 hogares; le sigue Chocó con 41.372 hogares; seguidamente Tolima y Caquetá con un reporte de 38.660 hogares y 38.552 hogares, respectivamente, y finalmente el departamento de Nariño quien reporta para este corte un total de 37.289 hogares. Los departamentos antes descritos, tuvieron la mayor concentración de las consecuencias del conflicto armado en el país con un total de $76 \%$ del desplazamiento. Los afectados por el desplazamiento como consecuencia del conflicto armado en Colombia, sufren, según Ramos et $\mathrm{al}^{13}$, una disminución importante de las fuentes de apoyo social que tienen disponibles dentro de la comunidad, que si bien es cierto, en algunos casos pueden lograr un desequilibrio negativo del bienestar psicosocial de cada afectado y como consecuencia directa agravar la situación de pobreza y exclusión social ${ }^{13}$, sin embargo, cabe anotar, que los desplazados en medio de su situación reciben más apoyo afectivo de los vecinos (personas de su comunidad) ${ }^{14}$ que de su misma familia.

Se podría asegurar que toda Colombia ha sido testigo de los actos de violencia como el desplazamiento que ha vivido la República por medios informativos o experiencias propias como las afectaciones que dejó la ola invernal en el 2010-2011, el "Fenómeno de la Niña", donde las lluvias han sido las más intensas y abundantes registradas en la historia del país, sobre todo en las regiones Caribe, Andina y Pacífica ${ }^{15}$ lo que causó desestabilización en las familias pertenecientes a comunidades ya establecidas. Los más afectados por el fenómeno invernal de nuestra investigación fueron aquellos habitantes que vivían cerca al río Magdalena catalogados en zona de alto riesgo, como plus a esta situación, sus ingresos son inferiores ${ }^{16,17}$ a un Salario Mínimo Mensual Vigente (SMMV).

Los departamentos que más necesitaron intervención por parte del gobierno por el Fenómeno de la Niña en el 20102011, estuvieron encabezado por Bolívar con 577.952; Magdalena con 337.048; Cauca con 333.392; Córdoba con 211.587; Atlántico con 175.839; Valle del Cauca con 172.807 y Cesar con $168.312^{18}$. Bolívar y Magdalena, con Cauca y Córdoba, en su orden, son los departamentos con mayor número de personas registradas, $29 \%$ del total. Le siguen Atlántico, Nariño, Antioquia, La Guajira, Valle del Cauca, Chocó, Sucre, Cesar y Tolima ${ }^{19}$.

La investigación considera que, para la población en estudio (victimas del desplazamiento forzoso y damnificados por la ola invernal), el gobierno, a través de sus políticas públicas, deben seguir fortaleciendo los programas enfocados en pro del bienestar y calidad de vida de los más afectados y sus familias dentro de su comunidad. Por eso, en esta investigación se pretendió establecer la correlación entre apoyo social y sentido de comunidad en personas en situación de desplazamiento por violencia y damnificados por la ola invernal del 20102011 en el departamento del Magdalena, Colombia, con el fin de realizar planes y programas de intervención para mejorar la dinamización comunitaria.

\section{MATERIALES Y MÉTODOS}

\section{Diseño}

Esta investigación se fundamenta en un paradigma hipotético-deductivo, bajo un diseño correlacional. De acuerdo con Hernández et $\mathrm{al}^{20}$, los estudios de tipo correlacional buscan evaluar la relación que puede existir entre dos o más variables en un único momento. Las investigaciones de este tipo, inician desde la individualidad de cada variable y luego la respectiva correlación entre ellas.

\section{Población}

Este estudio se realizó con 136 adultos, divididos en dos grupos, el primero estaba conformado por lo que sufrieron desplazamiento por la violencia política y los afectados por la ola invernal en el 2010-2011 y otro grupo que sólo padeció afectación de la ola invernal del 2010-2011 en el departamento del Magdalena.

Los participantes estuvieron distribuidos así:

1. (84) sujetos desplazados por violencia forzosa y afectados por la ola invernal 2010-2011.

2. (52) sujetos victimas sólo por la ola invernal 2010-2011. Los sujetos que participaron de esta investigación se seleccionaron por medio de un muestreo aleatorio a través del programa STATS y los criterios de inclusión tenidos en cuenta se relacionaban con que los participantes debían ser desplazados o damnificados, vivir en el Magdalena y haber vivido el/los evento/s en el mismo departamento y ser jefe de hogar o compañero(a) conyugal. 


\section{Instrumentos}

Para la caracterización de la población, y como valor agregado y tangencial a las pruebas, se aplicó un cuestionario sociodemográfico para obtener información de edad, género, estado civil, posición familiar, municipio donde viven, eventos sufridos, entre otros. De igual forma se utilizaron dos instrumentos: El primero, conocido como la escala de Sentido de Comunidad Índice SC-2 ${ }^{21}$, cuenta con 24 ítems en total y 4 áreas, cada una compuesta de 6 ítems. La SCI-2 mide las sub escalas como Integración/ Satisfacción \{1-2-3-4-5-6\}; Membresía \{7-8-9-10-11-12\}; Influencia \{13-14-15-16-17-18\} y Conexión Emocional $\{19-20-21-22-23-24\}$, la suma total del SCI es la sumatoria de la pregunta 1 a pregunta 26 y el segundo instrumento el Cuestionario de apoyo social-MOS ${ }^{22}$, cuenta con 20 ítems en total y 4 áreas, la primera Apoyo Social Emocional/ Informacional $\{3,4,8,9,13,16,17,19\}$, la segunda es Apoyo Instrumental $\{2,5,12,15\}$; la tercera Interacción Social Positiva $\{7,11,14,18\}$ y la cuarta es el Apoyo Afectivo $\{6,10,20\}$. Estos ítems son respondidos en una escala Likert de 5 opciones, con puntajes que van de 1 (nunca) a 5 (siempre). Además, el MOS cuenta con una pregunta de entrada: "Aproximadamente, ¿Cuántos amigos íntimos o familiares cercanos tiene Ud.?".

\section{Procedimiento}

Esta investigación fue dividida en cuatro fases: 1) Revisión de toda la bibliografía, se definieron las unidades de análisis y luego se procedió a la caracterización de la población escogida, seguidamente, se definió la muestra que se iba a intervenir por medio del programa STATS; 2) para la recolección de datos, se realizó una prueba piloto con 15 adultos en el municipio de Salamina, con su respectiva autorización y consentimiento informado con el objetivo de establecer la validez, comprensión y aprehensión de los participantes de la investigación, así como el tiempo de aplicación con cada adulto (promedio de 40 minutos aproximadamente). Luego, se aplicaron a 136 adultos las pruebas de Sentido de Comunidad y Apoyo Social; 3) para la tabulación y análisis de resultados, una vez que se recogió toda la información en campo (cuestionarios diligenciados por los participantes), se

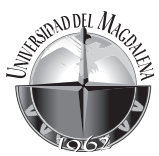

\section{Declaración de Aspectos Éticos}

Según la norma contemplada en la Resolución 008430 de 1993 del Ministerio de Salud ${ }^{23}$, ésta es una investigación de bajo riesgo. Se trabajó con desplazados por la violencia y damnificados por la ola invernal 2010-2011. Se utilizó un consentimiento informado de tipo escrito el cual firmaron voluntariamente. En esta investigación se establecieron las normas científicas, técnicas y administrativas para la investigación en salud, sin embargo, se aplicaron todas estas normas para la investigación en la comunidad, igualmente, estuvo sometida a lo dictaminado en la Ley $1090^{24}$. Como investigadores se respetó la confidencialidad de la información obtenida en el desarrollo de la investigación, se respetó la integridad y se protegió el bienestar de los sujetos.

\section{RESULTADOS}

Inicialmente en la investigación se aplicó un cuestionario sociodemográfico el cual arrojó que, en el primer grupo, los sujetos hombres $(\mathrm{n}=48)$ que participaron contaban con edades entre 26 y 89 años ( $=57,5$ años) y las sujetos mujeres $(n=36)$ contaban con edades entre 23 a 87 años ( $=55$ años). En el segundo grupo, los sujetos hombres $(\mathrm{n}=44)$ que participaron contaban con edades entre $25 \mathrm{y}$ 91 años ( $=58$ años) y las sujetos mujeres $(\mathrm{n}=8)$ contaban con edades entre 23 a 49 años ( $=36$ años), ambos grupos pertenecían a los municipios de Cerro de San Antonio, Tenerife, Salamina, El Piñón, Remolino y Zapayán, en el departamento del Magdalena.

En un primer momento se identificaron los índices de sentido de comunidad de los jefes de hogar que participaron en esta investigación, encontrando que el índice global de sentido de comunidad $(=53,42)$ es medio-alto, en cuanto a la pregunta inicial o introductoria sobre ¿Qué tan importante es tener un sentido de comunidad con otros miembros? se halló que es para la mayoría $(\mathrm{Mo}=6)$ era "Muy importante". En cuanto a la integración/satisfacción de la población que se estudió se halló que existe un nivel medio-alto $(=13,47)$ de esta sub variable entendida como esos beneficios que tiene cada sujeto integrante de una comunidad que recibe como retroalimentación por pertenecer a ésta en término de estatus, valores, respeto y apoyo psicológico en momentos de necesidad ${ }^{25}$. En cuanto a la membresía también existe un nivel medio-alto $(=11,72)$. Los sujetos estudiados presentan un nivel de influencia medio-alto $(=13,02)$ 
lo que es considerado como esa capacidad percibida que tiene un sujeto para ser influenciado por un grupo o el grupo sea influenciado por la comunidad o ésta influya sobre otros grupos ${ }^{26}$; y finalmente, al evaluar la sub escala de conexión emocional se halló que existe $(=15,21)$ un nivel alto dando mayor sostenibilidad y empoderamiento al sentido de comunidad. La conexión emocional permite interacción fluida en la comunidad entre sus miembros ${ }^{26}$. La conexión emocional es el sentimiento de puede lograr una persona o un grupo de personas de hacer parte de una comunidad, por medio de los eventos sociales como fechas fundamentales, apodos o sobrenombres, amistades, elecciones políticas y demás actividades que pueden compartir juntos en todo un conjunto de proyecto de vida de cada ser humano que tiene vínculos afectivos o emocionales directo con la comunidad. Se podría considerar que la conexión emocional, es el componente clave y fundamental que se da en el proceso de construcción del Sentido de Comunidad26,27 (Tabla1).

Tabla 1. Percepción de Sentido de Comunidad

\begin{tabular}{|l|c|c|c|c|c|c|c|}
\hline & Media & \multirow{2}{*}{ Mediana } & \multirow{2}{*}{ Moda } & \multirow{2}{*}{$\begin{array}{c}\text { Desviación } \\
\text { estándar }\end{array}$} & \multicolumn{3}{|c|}{ Percentiles } \\
\cline { 6 - 9 } & & & & & 25 & 50 & 75 \\
\hline Total Del ISC & 53,42 & 53,00 & 46 & 8,741 & 47,00 & 53,00 & 59,00 \\
\hline $\begin{array}{l}\text { iQué tan importante } \\
\text { es para usted sentir un } \\
\text { sentido de Comunidad? }\end{array}$ & 5,74 & 6,00 & 6 &, 439 & 5,00 & 6,00 & 6,00 \\
\hline Integración/ satisfacción & 13,47 & 13,00 & 11 & 2,794 & 11,00 & 13,00 & 16,00 \\
\hline Membresía & 11,72 & 11,00 & 11 & 2,532 & 10,00 & 11,00 & 13,75 \\
\hline Influencia & 13,02 & 13,00 & 11 & 2,882 & 11,00 & 13,00 & 15,00 \\
\hline Conexión emocional & 15,21 & 16,00 & 18 & 2,251 & 13,00 & 16,00 & 17,00 \\
\hline
\end{tabular}

En un segundo momento se evaluó el apoyo social en las personas en situación de desplazamiento por la violencia y damnificados por la ola invernal del 20102011 en el departamento del Magdalena, encontrando que la percepción del apoyo social de forma general es alto $(=87,41)$, es decir, presenta niveles altos de apoyo emocional/informacional, apoyo instrumental, tiene una excelente interacción social y tienen apoyo afectivo, lo dicho hasta aquí, supone que el apoyo social es un proceso complejo que implica de manera directa la interacción entre las estructuras sociales, las relaciones sociales y los atributos personales (Tabla 2).

Tabla 2. Percepción de Apoyo Social

\begin{tabular}{|c|c|c|c|c|c|c|c|}
\hline & Media & Mediana & \multirow{2}{*}{ Moda } & Desviación & \multicolumn{3}{|c|}{ Percentiles } \\
\cline { 6 - 8 } & & & & estándar & 25 & 50 & 75 \\
\hline $\begin{array}{c}\text { Índice global de apoyo } \\
\text { social }\end{array}$ & 87,41 & 88,00 & 95 & 6,952 & 82,00 & 88,00 & 95,00 \\
\hline Apoyo social emocional & 36,24 & 37,00 & 40 & 3,575 & 33,00 & 37,00 & 40,00 \\
\hline Apoyo instrumental & 19,01 & 20,00 & 20 & 1,488 & 18,00 & 20,00 & 20,00 \\
\hline Interacción social positiva & 18,09 & 18,00 & 20 & 1,880 & 17,00 & 18,00 & 20,00 \\
\hline Apoyo afectivo & 14,07 & 15,00 & 15 & 1,184 & 13,00 & 15,00 & 15,00 \\
\hline
\end{tabular}


En un tercer momento se determinó que existe una relación débil entre el Índice General de Apoyo Social y el Índice General de Sentido de Comunidad $(\mathrm{r}=0.269)$ y significativa (Valor P de 0,13 ), ante esto, los investigadores podrían decir que es muy probable que, sin importar haber sufrido dos eventos traumáticos, los afectados podrían tener más sentido de comunidad y apoyo social a diferencia de los que no hayan sufrido eventualidades conjuntas.
Para el caso de los damnificados de la ola invernal, la relación es muy baja $(0,158)$ y no significativa (Valor $P$ de 0,262), hecho que permite creer que no hay una relación entre las variables analizadas en el caso de afectación sólo por un evento como ola invernal, lo que traduce que para los damnificados por la ola invernal en el departamento del Magdalena no existe una clara relación entre las dos variables de sentido de comunidad y apoyo social (Tabla 3).

Tabla 3. Correlaciones de las variables Apoyo Social y Sentido de Comunidad en los dos grupos

\begin{tabular}{|c|c|c|c|}
\hline \multirow{2}{*}{ Correlación } & SENTIDO DE COMUNIDAD \\
\hline \multirow{4}{*}{ APOYO SOCIAL } & \multirow{2}{*}{$\begin{array}{c}\text { Damnificados Y } \\
\text { Desplazados }\end{array}$} & $\begin{array}{c}\text { Correlación de } \\
\text { Pearson }\end{array}$ &, $269^{*}$ \\
\cline { 3 - 4 } & \multirow{3}{*}{ Damnificados } & Sig. (bilateral) &, 013 \\
\cline { 3 - 4 } & & $\mathrm{N}$ & 84 \\
\cline { 3 - 4 } & & $\begin{array}{c}\text { Correlación de } \\
\text { Pearson }\end{array}$ &, 158 \\
\cline { 3 - 4 } & & Sig. (bilateral) &, 262 \\
\hline
\end{tabular}

*. La correlación es significativa en el nivel 0,05 (2 colas).

\section{DISCUSIÓN}

Los sujetos víctimas del conflicto armado y damnificados por la ola invernal estudiados en esta investigación realizada en el departamento del Magdalena de manera general presentan un nivel medio-alto de sentido de comunidad, que se entiende como la forma positiva que las personas logren integrarse mejor a su comunidad y por ende los sujetos logran involucrarse más en iniciativas de desarrollo comunitario ${ }^{28,29,13}$, demostrando los sentimiento de pertenecer a un grupo establecido y demostrar que los miembros importan los unos a los otros ${ }^{28}$.

El Índice de Apoyo Social para estos sujetos fue alto, lo que demuestra que la población en general percibe apoyo social dentro de su comunidad, inicialmente de sus amigos y familiares ${ }^{30}$ lo que genera sentimiento de seguridad ${ }^{31,2}$ puesto que existen lazos fuertes ${ }^{32}$ que permiten acceder de manera directa a ese apoyo social percibido en todo el contexto social, en donde se brindará apoyo en solución y afrontamiento de problemas, muestras de empatía, consideración, amor, lealtad, sinceridad y confianza ${ }^{33}$, entre otras, que favorecerán el apoyo social entre los sujetos de la comunidad.

El apoyo social se expresa en empatía, amor y confianza $^{31,33,34}$. A través de la interacción social positiva, los individuos y la comunidad constituyen una fuente de apoyo vital, de donde se derivan sentimientos de pertenencia con importantes implicaciones transversales para el bienestar individual y social, lo que generaría como efectos psicológicos positivos en las personas, cuando existe esa reciprocidad del apoyo social ${ }^{35}$.

Para el grupo de desplazados por la violencia y damnificados por la ola invernal existe una relación débil entre el Índice General de Apoyo Social y el Índice General de Sentido de Comunidad. En un estudio realizado en Almería-España, se centró en el análisis de convergencia entre dos variables apoyo social y bienestar subjetivo en un grupo de inmigrantes marroquíes que son residentes de Almería. En esta investigación se halló que el apoyo social que reciben estos sujetos por sus relaciones interpersonales en su comunidad de forma general era 
satisfactorio, en su mayoría el $53 \%$ de la población que se estudió expresaba un alto grado de satisfacción en apoyo social; y el restante de la población $47 \%$ se muestra moderadamente satisfechas, concluyendo que no existía rasgos de insatisfacción por los sujetos estudiados ${ }^{36}$.

En la taxonomía del apoyo social, Gracia y Herrero ${ }^{37}$ incluyen la integración social como ese constructo que hace referencia a la participación e implicación que tiene una persona en la vida social dentro de su comunidad y por ende de la sociedad de donde pertenece. Ligado a este orden, Prezza et $\mathrm{al}^{38}$, muestran los resultados donde el sentido de comunidad aparece relacionado de manera directa e implícita en establecer relaciones íntimas dentro de la comunidad como amistades o alianzas matrimoniales, tiempo de convivir en la comunidad, participación activa en reuniones, grupos especiales y otras que sean predictores del sentido de comunidad ${ }^{39}$. En la misma línea, se encontró que si lo sujetos tienen un mayor lapso en el barrio, mayor y fuerte va hacer las relaciones con los vecinos y como consecuencia incide en un aumento del sentido de comunidad ${ }^{40}$. En la población estudiada, bajo el tiempo estipulado de la investigación (alrededor de 15 meses) se confirmó que el sentido de comunidad efectivamente converge con las variables que explica Prezza et al ${ }^{38}$, además es importante recalcar que no toda la población afectada por los eventos (desplazamiento o Fenómeno de la Niña) salieron de sus viviendas, un porcentaje expresaban $(10 \%)$ que de sus viviendas no se movían porque no se sentían con garantía que el gobierno les ayuda a superar esta crisis.

El apoyo social juega un papel importante en las personas que sufrieron eventos traumáticos ya que éste, de forma positiva, promueve la salud y el bienestar y ayuda a reducir reacciones negativas ante el estrés ${ }^{41}$.

Para el caso de los damnificados de la ola invernal, la relación es muy baja, se considera que los sujetos que participaron en la investigación que sufrieron ambos eventos traumáticos presentan una relación leve entre sentido de comunidad mayor y apoyo social eficaz, contrario a aquellos que solo sufrieron un solo evento como la ola invernal. Se puede deducir que el hecho de haber sufrido dos eventos traumáticos como el desplazamiento y luego la ola invernal, hacen que los sujetos de este grupo $(\mathrm{N}=84)$ tengan desarrollada más la capacidad de resiliencia que aquellos que sólo han sufrido un solo evento $(n=52)$, en otras palabras, existe una relación leve entre sentido de comunidad y apoyo social en los sujetos que han pasado por dos eventos traumáticos en el departamento del Magdalena como ha sido el desplazamiento por la violencia forzosa y eventos naturales como la ola invernal 2010-2011 contario al grupo que sólo sufrió una afectación.

La cuestión se engloba que los afectados por los dos acontecimientos, al parecer, tienen un mayor potencial humano lleno de esperanza y responsabilidad colectiva en la promoción del cambio social ${ }^{42,43}$ lo que llama a una transformación de ayuda mutua entre los afectados.

Estas nociones se pueden fundamentar bajo la investigación realizada en la Universidad de Talca en 2011, sobre el la resiliencia, apoyo social e impacto del terremoto y tsunami del 27-f en estudiantes donde se encontró que existe una correlación positiva entre la percepción de apoyo social y resiliencia, en este mismo estudio se constató que las personas que tuvieron daños familiares presentaron mayor percepción de apoyo social ${ }^{44}$, al igual que en Colombia, la causa del desplazamiento o eventos naturales dejan todo tipo de daños ya sean familiares o de infraestructuras ${ }^{15,45}$. En esta misma línea, se demostró en la investigación realizada en los barrios de Málaga-España en el 2010 que existe una relación positiva entre sentido de comunidad y satisfacción con la calidad de vida de la población, lo que confirma la relación positiva entre sentido de comunidad y calidad de vida, siendo el sentido de comunidad un elemento clave y fortalecedor de la interacción social ${ }^{46}$.

La relación existente entre estos dos índices (Sentido de comunidad y Apoyo Social) es débil o baja y positiva con un coeficiente de correlación de $(\mathrm{r}=0,278)$ y un valor ( $\mathrm{P}$ de 0,01$)$ lo que permite creer que la relación existente es significativa. La relación existente entre las dos variables no permite una explicación precisa sobre los efectos que tiene el apoyo social sobre el sentido de comunidad o viceversa, conviene subrayar que la relación entre estas variables sea inexistente, solo que es más difícil asignar algún tipo de efecto entre las variables.

\section{CONCLUSIÓN}

A partir de los resultados, se puede deducir que los sujetos de la investigación que pasaron por eventos como el desplazamiento forzado y los que pasaron por eventos naturales como la ola invernal del 2010-2011 en los municipios del departamento del Magdalena, al Norte de la República de Colombia, fueron situaciones que generaron desestabilidad a una comunidad conformada.

Dentro del análisis descriptivo para la variable de sentido de comunidad, se descubrió que ésta de forma general, está en un nivel medio-alto, esto es, que la población 
estudiada están satisfechos con sus comunidades (municipios donde viven), ellos tienen sentimientos de respeto, amor, sentido de pertenencia, además, los sujetos en general, tienen un nivel alto de conexión emocional lo que permite que la colectividad mayor presente entre ellos una red sólida de relaciones donde el apoyo mutuo sea la esencia de la comunidad.

En cuanto a la variable de apoyo social, se halló que los sujetos en la investigación refieren tener apoyo social en un nivel alto, entendiéndolo como la obtención del bienestar afectivo en un grupo que soluciona los problemas que más lo aquejan, la relación o interacción entre ellos es excelente o positiva, garantizando su estabilidad como grupo comunitario mejorando.

Aunque se presenten Sentido de Comunidad y Apoyo Social en la comunidad de forma individual, al momento de hacer el análisis correlacional se descubrió que la relación entre las dos variables es débil o baja de forma general, pero significativa. Dentro de lo que es significativo, se halló que los sujetos pertenecientes al departamento del Magdalena tienen Sentido de Comunidad por los lugares donde viven, aunque ahí hayan pasado los dos eventos traumáticos o uno sólo y el apoyo social lo perciben en niveles altos, lo que representaría que son sujetos activos dentro de su comunidad que brindan apoyo a sus vecinos.

Se concluye que esta investigación con los desplazados por violencia forzosa y damnificados por la ola invernal da un aporte valioso a los grupos de investigaciones e instituciones gubernamentales y no gubernamentales principalmente en el departamento del Magdalena, puesto que a través de los resultados de los índice de Sentido de Comunidad y Apoyo social tanto en desplazados por violencia forzosa y damnificados de la ola invernal 2010-2011 fueron altos relativamente desde el análisis descriptivo, se podría enriquecer a futuro, añadiendo otras variables como medición de resiliencia, calidad de vida u otra que pueda conectarse con esta investigación. Habría que decir también, que los resultados conseguidos en esta investigación en el departamento del Magdalena llegarían a ser un valioso insumo para que las organizaciones creen programas de intervención comunitaria a favor de los afectados por el desplazados por violencia forzosa y damnificados por la ola invernal 2010-2011 con la finalidad de mejorar la dinamización comunitaria, además, será un gran aporte al conocimiento, para fortalecer los estudios en este campo en la región Caribe, referente a las variables estudiadas en la investigación.
Los eventos como desplazamiento forzoso o eventos naturales, como el Fenómeno de la Niña, causan algún tipo de impacto a los afectados, pero es en esas situaciones donde la mayoría de personas activan su capacidad de resiliencia para avanzar antes las adversidades y les ayudará a empezar nuevamente a construir su proyecto de vida personal y familiar. Según el tipo de personalidad de cada ser humano, las adversidades son tomadas como un fracaso y/o nuevas oportunidades que les da la vida para afrontar situaciones que se le salen de sus manos.

\section{DECLARACIÓN SOBRE CONFLICTOS DE INTERESES}

Los autores no reportan conflictos a declarar relacionados con la investigación.

\section{REFERENCIAS BIBLIOGRÁFICAS}

1. Watanabe C, Okumura J, Chiu T, Wakai S. Social support and depressive symptoms among displaced older adults following the 1999 Taiwan earthquake. Journal of Traumatic Stress. 2004;17(1):63-7.

2. Lin N, Dean A, Ensel W. Social support, life events and depression. London: Academic Prees; 1986.

3. Sarason S. The psychological sense of community: prospects for a community psychology. Washington: Iberoamericana; 1974.

4. Távara M. Sentido de Comunidad en un contexto de violencia comunitaria. [Tesis maestría en Psicología Comunitaria con mención en Salud Mental]. Perú: Pontificia Universidad Católica del Perú; 2012.

5. Brodsky A. Resilient single mothers in risky neighborhoods: Negative psychological sense of community. Journal of Community Psychology. 1996 Jan; 24(4): 347-363.

6. Mendoza A. El desplazamiento forzado en Colombia y la intervención del Estado. Revista de Economía institucional [revista en la Internet]. 2012 [citado 2015 May 12]; 14(26):169-202. Disponible en: http://www. economiainstitucional.com/pdf/no26/amendoza.pdf

7. Murad R. Estudio sobre la distribución espacial de la población de Colombia. Serie Población y Desarrollo. CEPAL. Colombia; 2003.

8. Colombia. Ley 387 de 1997 por la cual se dictan las medidas para la prevención del desplazamiento forzado; la atención, protección, consolidación y esta estabilización socioeconómica de los desplazados internos por la violencia en la República de Colombia. Diario Oficial, 43091 (Jul. 24 1997). 
9. Colombia. Ley 1448 de 2011 por la cual se dictan medidas de atención, asistencia y reparación integral a las víctimas del conflicto armado interno y se dictan otras disposiciones. Diario Oficial, 48096 (Jun 10 2011).

10. Mora A. Conflicto, Violencia Socioeconómica Y Desplazamiento Forzado En Colombia. Cuadernos de Economía [revista en la Internet]. 2013 [citado 2015 Feb 10]; 32(61): 721-754. Disponible en http://www. revistas.unal.edu.co/index.php/ceconomia/article/ view/42495/45956.

11. Colombia. Unidad de Victimas. Registro Único de Victimas. Colombia: UARIV; 2011.

12. Colombia. Departamento para la Prosperidad Social. Desplazamiento Forzado en Colombia. Colombia: DPS; 2010.

13. Ramos I, Holgado D, Maya I. Las redes personales de los desplazados internos por la violencia política en Colombia: una aproximación al caso del departamento del Atlántico. Trace [revista en la Internet]. 2014 [citado Jun 13]; (65): 51-68. Disponible en: http://www.scielo. org.mx/pdf/trace/n65/n65a5.pdf

14. Palacio J, Madariaga C. Lazos predominantes en las redes sociales personales de desplazados por violencia política. Investigación y desarrollo [revista en la Internet]. 2006 [citado 2015 Feb 6]; 14 (1): 86-119. Disponible en: http://rcientificas.uninorte.edu.co/index. php/investigacion/article/viewFile/945/567.

15. Portafolio Fondo Adaptación. Atención a víctimas del llamado "Fenómeno de la Niña". Colombia: FA; 2013.

16. Kahn, M. The death toll from natural disasters: the role of income, geography, and institutions. Review of Economics and Statistics [revista en la internet]. 2003 [citado 2015 Mayo 15]; 87(2): 271-84. Disponible en: http://earthmind.org/files/risk/Tufts-2003-Income-GeoInstitutions-Role-in-DRR.pdf

17. Palacio J, Barrios B. Relación entre las estrategias de afrontamiento colectivo y el crecimiento postraumático en adolescentes damnificados por la ola invernal. Psicogente [revista en la Internet]. 2013 [citado 2015 Jun 14]; 16(30):345-355. Disponible en: http:// publicaciones.unisimonbolivar.edu.co/rdigital/ojs/ index.php/psicogente/article/view/284/281

18. Colombia. Alta Consejería para las Regiones y la Participación Ciudadana. Reporte Fenómeno de la Niña en Colombia. Colombia; 2012.

19. Comisión Económica para América Latina y el Caribe - CEPAL. Valoración de daños y pérdidas. Ola invernal en Colombia, 2010-2011. Bogotá: Misión BID - CEPAL; 2012. [Internet] [citado 2015 Jun 28]. Disponible en: http://www.cepal.org/publicaciones/xml/0/47330/ olainvernalcolombia2010-2011.pdf

20. Hernández R, Fernández C, Baptista P. Metodología de la investigación. Quinta edición. Editorial Mc Graw Hill; 2010.
21. Chavis D, Lee K, Acosta J. The Sense of Community (SCI) Revised: The Reliability and Validity of the SCI-2. En: 2nd International Community Psychology Conference; Lisboa 2008 Juni 6-8. Lisboa; 2008.

22. Londoño AN, Rogers HL, Castilla TJ, Posada GS, Ochoa AN, Jaramillo PM et al Validación en Colombia del cuestionario MOS de apoyo social. Int. j. psychol. res. (Colombia). 2012; 5(1):142-150.

23. Colombia. Cundinamarca. Ministerio de Salud. Resolución 008430 de 1993, octubre 4, por la cual se establecen las normas científicas técnicas, y administrativas para la investigación en salud. Bogotá: Ministerio de Salud;1993.

24. Colombia. Ministerio de la Protección Social. Ley 1090 de 2006 por la cual se reglamenta el ejercicio de la profesión de Psicología, se dicta el Código Deontológico y Bioético y otras disposiciones. Diario Oficial, 46383 (Sep 62006 )

25. Montero M. Introducción a la psicología comunitaria. Desarrollo, conceptos y procesos. Primera edición. Buenos Aires: Editorial Paidós; 2004.

26. Fernández I, Morales J, Molero F, coordinadores. Sentido de Comunidad. Psicología de la Intervención Comunitaria. España: Desclée de Brouwer; 2011:97-128.

27. McMillan D, Chavis D. Sense of community: a definition and theory. Journal of Community Psychology. 1986 Jan; 14;6-24.

28. Chavis D, Wandersman A. Sense of community in the urban environment: a catalyst for participation and community development. American Journal of Community Psychology. 1990 Feb; 18(1): 55-8.

29. Kloos B, Hill J, Thomas E, Wandersman A, Elias M, Dalton, J. Community psychology: Linking individuals and communities. Third Edition [Internet]. Wadsworth. Cengage Learning; 2012 [citado 2015 Abr 04]. Disponible en: https://books.google.com.co/books?hl = es\&lr = \&id $=8 \mathrm{XOKAAAAQBAJ} \& \mathrm{oi}=$ fnd $\& \mathrm{pg}=\mathrm{PR} 5 \& \mathrm{dq}=$ Communit y + psychology: + Linking + individuals + and + commun ities\&ots $=$ nwz7d_94sY\&sig $=$ Pi-cr9OHzUlmmHGEYlh$8 \mathrm{zm} 6 \mathrm{vj} 2 \mathrm{I} \# \mathrm{v}=$ onepage $\& \mathrm{q}=$ Community $\% 20$ psychology $\% 3 \mathrm{~A} \% 20$ Linking $\%$ 20individuals $\% 20$ and $\% 20$ communities\&f $=$ false

30. House J. Work Stress and Social Support. Reading: Addison-Wesley;1981.

31. Gottlieb B. Social support strategies: Guidelines for mental health practice. Beverly Hills: Sage Publications; 1983.

32. Granovetter M. The strength of weak ties. American Journal of Sociology. 1973; 78(6): 1360-1380.

33. Vega O, González D. Apoyo social: Elemento clave en el afrontamiento de la enfermedad crónica. Enfermería Global [revista en la Internet]. 2009. [citado 2015 Feb 05];(16):1-11. Disponible en: file://C:/Users/ Marina \% 20Camargo/Downloads/66351-277121-1PB.pdf. 
34. Antonucci T, Israel B. Veridicality of social support: A comparison of principal and network member's responses. Clinical Psychology. 1986;54(4): 432-437.

35. Cohen S, Gottlieb B, Underwood L. Social relationships and health. En: Cohen S, Underwood L, Gottlieb B. Social support measurement and intervention: A guide for health and social scientists. New York: Oxford University Press; 2000. p. 3-25.

36. Hernández S, Pozo C, Alonso E. Apoyo Social Y Bienestar Subjetivo En Un Colectivo De Inmigrantes ¿Efectos directos o amortiguadores?. Boletín de Psicología [revista en la Internet]. 2004 [citado 2015 Marz 05];(80);79-96. Disponible en: http://www.uv.es/ seoane/boletin/previos/N80-4.pdf

37. Gracia E, Herrero J. La Comunidad como Fuente de Apoyo Social: Evaluación e Implicaciones en los Ámbitos Individuales y Comunitarios. Revista Latinoamericana de Psicología [revista en la Internet]. 2006 [citado en 2015 Feb 10]; 38(2):327-342. Disponible en: http://www. scielo.org.co/pdf/rlps/v38n2/v38n2a07.pdf

38. Prezza M, Amici M, Roberti T, Tedeschi G. Sense of community referred to the whole town: Its relations with neighboring, loneliness, life satisfaction, and area of residence. Journal of Community Psychology. 2001 Jan; 29(1): 29-52.

39. Chipuer H, Pretty G. A review of sense of community index: Current uses, factor structure, reliability and further development. Journal of Community Psychology.1999 Nov; 27(6): 643-658.

40. Robinson D, Wilkinson D. Sense of Community in a Remote Mining Town: Validating a Neighborhood
Cohesion Scale. T. American Journal of Psychology. 1995 Feb; 23(1): 137-48

41. Cutrona C, Hessling R, Suhr J. The influence of husband and wife personality on marital social support interactions. Personal Relationships.1997 Dec; 4(4): 379-93.

42. López V. Educación y resiliencia: alas de la transformación social. Actualidades Investigativas en Educación [revista en la Internet]. 2010 [citado 2015 Abr 5]; 10 (2): 1-14. Disponible en: https://e-nautia.com/portafolio/ disk/DOCENTE/MATERIAL \% 20HIPOTESIS \% 20 ACCION/RESILIENCIA \% 20/RESILIENCIA \% 20Y \% 20 EDUCACION.pdf

43. Amar JJ, Madariaga C, Jabba D, Abello R, Palacio JE, De Castro A et al Desplazamiento Climático y Resiliencia. Uninorte (Barranquilla).2014;1-146.

44. Alarcon S, Portius D, Méndez M. Resiliencia, apoyo social e impacto del terremoto y tsunami del 27-f en estudiantes de educación básica de la ciudad de constitución [Trabajo de grado en Psicología]. Chile: Universidad de Talca. Facultad de Psicología; 2011.

45. Consultoría para los Derechos Humanos y el Desplazamiento. Víctimas emergentes - Desplazamiento, derechos humanos y conflicto armado. Bogotá: CODHES; 2009.

46. Hombrados M. Calidad de vida y Sentido de Comunidad en la ciudad. Uciencia [revista en la Internet]. 2010 [citado 2015 Marz 15];(3):38-41. Disponible en: http://dspace. uma.es/xmlui/bitstream/handle/10630/4057/38_ revistauciencia03.pdf? sequence $=1$

Para citar este artículo: Camargo-Velásquez A, Palacio-Sañudo J. Apoyo social y sentido de comunidad en desplazados y damnificados en el departamento del Magdalena. Duazary. 2017 enero; 14 (1): 25 - 44 